\title{
Privacy-Preserving Discovery of Topic-Based Events from Social Sensor Signals: An Experimental Study on Twitter
}

\author{
Duc T. Nguyen and Jai E. Jung \\ Department of Computer Engineering, Yeungnam University, Gyeongsan 712-749, Republic of Korea \\ Correspondence should be addressed to Jai E. Jung; ontology.society@gmail.com
}

Received 22 January 2014; Accepted 10 February 2014; Published 3 April 2014

Academic Editor: Jason J. Jung

Copyright ( 2014 D. T. Nguyen and J. E. Jung. This is an open access article distributed under the Creative Commons Attribution License, which permits unrestricted use, distribution, and reproduction in any medium, provided the original work is properly cited.

\begin{abstract}
Social network services (e.g., Twitter and Facebook) can be regarded as social sensors which can capture a number of events in the society. Particularly, in terms of time and space, various smart devices have improved the accessibility to the social network services. In this paper, we present a social software platform to detect a number of meaningful events from information diffusion patterns on such social network services. The most important feature is to process the social sensor signal for understanding social events and to support users to share relevant information along the social links. The platform has been applied to fetch and cluster tweets from Twitter into relevant categories to reveal hot topics.
\end{abstract}

\section{Introduction}

Online services have been playing an important role in human life. Since these services help people to connect, transmit, share, and support diverse demands, most of people are using these services at work and daily activities. In particular, social network service (called SNS) has become more popular than the traditional communication media, for example, e-mail, SNS, video streams, and so on. The wellknown SNSs are as follows ("How many people use the top social media Apps \& Services" by Craig Smith, September 2013, on Digital Marketing Ramblings): Twitter (over 500 million users, more than 200 million active users), Facebook (1.15 billion users), Flickr (over 87 million users, 8 billion photos are shared), YouTube (over 1 billion users, 4 billion views per day), and so on.

These SNSs can be used with a number of types of social relationships, for example, a group of friends, a community of like-minded users, or business company and their customers. The users can share or announce their ideas, activities, and interesting events with others. Possible reasons are broad connection, diversity of applications, OS-independence, ease of use, and explosion in the number and kind of personal devices which are integrated wireless components, for example, smart phone, cameras, camcorders, and so on. It allows people to quickly update the latest information, their own opinion, or commentary toward a daily event. It opens a challenge issue of detecting which conversation topic trends are discussed and how to cluster incoming messages into relevant topic categories.

For example, many news organizations use Twitter daily to post new short message with the purpose of sending information to a wide variety of people about hot news. A combination of information from multiple sources can show a completed image about one or a group of facts. Particularly, if it is interesting, more commentary and sharing action will be done by SNS's members. However, because of huge number of messages, it is difficult for people to follow all news and also to know what the significant information is. So, Figure 1 shows an example about a demand of tracking relevant message; we need an application that can notify what is discussed on SNSs right after these messages appeared on the data stream. And it is very useful if the application can extract embedded information in its content that will help to reduce time of monitoring and managing news on social networks.

With assumption that a social topic or an event will attract more attention from people when and around its first time of occurrence either in public or among a group of related users such as hobby group, technology community, and business 


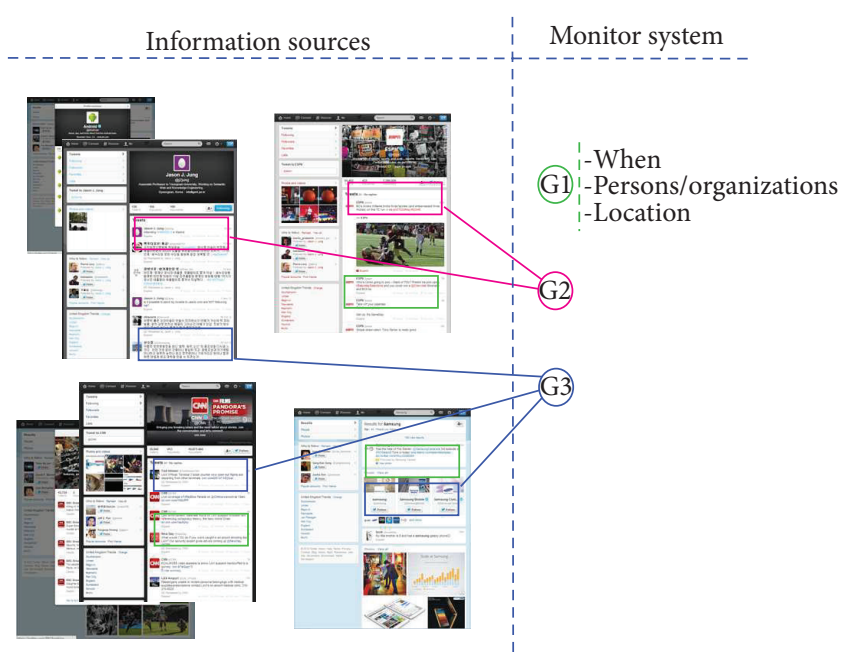

Figure 1: Example about demand to monitor and aggregate information.

network. Its signal can be expressed at the rapid increase in a short time range of

(1) the number of messages described about a fact even if it is the personal opinions;

(2) the response transactions which includes replying, discussion, or sharing action on an original message;

(3) the frequency occurring of terms including meaningful keywords, named entities, or phrases.

In this paper, we only discuss topics, which relate to a community or to public scale, gathering a large enough number of messages about their context. These messages are collected in runtime on a data pipeline from Stream API (application programming interface) of SNS sites; the number of incoming message at a certain time can be large so that it requires an effective method to be processed, especially, when we use the rapidly changing symptoms of occurring frequency terms and the cooccurring frequency between them to look for topic trend candidates.

The outline of this paper is organized as follows. In Section 1, we introduce the problem and our approach of event detection and event-based clustering, in general; other related works are presented in Section 2; Section 3 shows an approach to build a system for monitoring and detecting topic trend; at the end, we show our experimental result in Section 4 and draw the conclusion and the future work in Section 5.

\section{Related Work}

In this work we deal with the problem of topic trend detection. Also, many researchers have tried to deal with the same problem in previous work [1-9]. Just like our social networks in real world, online social network is a complex organization that reflects participants' relationships in an open and almost unlimited environment to connect people around the world [10-12]. The number of posting

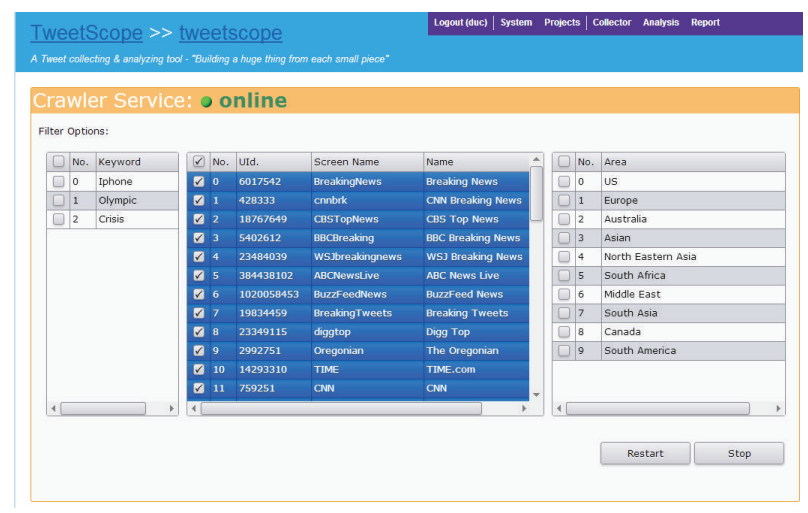

FIGURE 2: Example about demand to monitor and aggregate information.

messages on SNS is increasing day-by-day with the growth of computer, web application, and personal smart devices. It raises a challenge of analyzing and determining real-time and large-scale data for many studies from technical works to business applications $[13,14]$. Most related researches to this work are about the problem of topic trend detection and clustering in social network where incoming messages are treated as a data stream $[1,2,4,8,10,15]$; some works consider the incoming messages as the time series to track event by looking for changing data points $[5,6,16]$.

\section{Topic Detection and Clustering}

We consider that SNS is a complex structure where people connect each each other via their relationships (e.g., friendship). Along these social structures, the information is sequentially transmitted from one to the other. This transmission sequence can be regarded as an instance of time series, where each information package is a data point with fully information for the next analysis phases.

3.1. Data Crawler. The data stream is temporal; it contains some messages which are fully embedded with rich information about facts or topic trends. We assume that whenever a topic attracts attention of people, a lot of relevant messages will be posted in a short time range around its appearance time. Overtime, a topic trend will appear and disappear depending on its novelty and attractive content, so we call topics life cycle an approximate time range from a timestamp when people start to discuss something to the time when people do not talk about it so much. It needs an application which can work as an online application to fetch and classify incoming message as quick as possible, so that data service and web-based techniques are suitable to implement it. Figure 2 is an illustration of the application GUI to manage the crawling functions, where data sources are selected from Twitter accounts of famous breaking news agent (http://twitaholic.com-Tool of tracking the most popular users of a certain microblogging/social network).

We discretize the data stream by using a sampling function with an interval $\Delta t$; besides that depending on 
TABLE 1: List of detected events from BND data set.

\begin{tabular}{|c|c|c|c|c|}
\hline \multirow{2}{*}{ Number } & \multirow{2}{*}{ Related events } & \multicolumn{2}{|c|}{ Event date (GMT) } & \multirow{2}{*}{ Topic terms } \\
\hline & & Approximate date & Detected time & \\
\hline 1 & Ricin letter is sent in US & April 18, 2013 & April 18, 2013 00:41 & Containing FBI, justice, letters, ricin, sent \\
\hline 2 & $\begin{array}{l}\text { fighters from PKK Kurdish separatist } \\
\text { group leaving Turkey }\end{array}$ & April 25, 2013 & April 25, 2013 13:15 & $\begin{array}{l}\text { fighters, Iraq, Kurdish, leaving, pkk, } \\
\text { turkey }\end{array}$ \\
\hline 3 & $\begin{array}{l}\text { Boeing } 787 \text { Dreamliner arrives safely at } \\
\text { Nairobi in the first commercial flight }\end{array}$ & April 27,2013 & April 27,201307:55 & $\begin{array}{l}\text { The first, 787, Boeing, commercial, } \\
\text { Dreamliner, flight }\end{array}$ \\
\hline 4 & Bomb car attacks in Baghdad & May 27, 2013 & May 27, 2013 15:11 & $\begin{array}{l}\text { Attacks, Baghdad, bombs, car, mainly, } \\
\text { Shia }\end{array}$ \\
\hline 5 & Protest in Turkey & May 31, 2013 & May 31, 2013 15:05 & Plans, square, Taksim \\
\hline 6 & North Korea proposes talk with the USA & June 6, 2013 & June 6, 2013 10:54 & Korea, north, proposes, talks \\
\hline 7 & $\begin{array}{l}\text { Turkey using tear gas and water cannon } \\
\text { again protesters }\end{array}$ & June 11, 2013 & June 11, 2013 17:26 & $\begin{array}{l}\text { Cannon, enter, fired, gas, Gezi, Istanbul, } \\
\text { park, protesters, tear, trying, water }\end{array}$ \\
\hline 8 & Nelson Mandela’s death & June 11, 2013 & June 11, 2013 22:10 & $\begin{array}{l}\text { African, hospital, Mandela, Nelson, } \\
\text { critical, improvement, stable }\end{array}$ \\
\hline 9 & $\begin{array}{l}\text { Lionel Messi and tax law infringement } \\
\text { issue }\end{array}$ & June 12, 2013 & June 12, 2013 13:14 & $\begin{array}{l}4 \mathrm{~m}, 5 \mathrm{~m} \text {, euros, father, filed, fraudulent, } \\
\text { Lionel, Messi, returns, Spain, tax }\end{array}$ \\
\hline 10 & $\begin{array}{l}\text { Edward Snowden takes a flight to } \\
\text { Moscow Airport }\end{array}$ & June 23, 2013 & June 23, 2013 16:42 & Asylum, Edward, Snowden, tweets \\
\hline 11 & $\begin{array}{l}\text { Snowden is in transit area of Moscow } \\
\text { airport }\end{array}$ & June 25, 2013 & June 25, 2013 14:49 & $\begin{array}{l}\text { Airport, confirms, fugitive, president, } \\
\text { Snowden, transit }\end{array}$ \\
\hline 12 & Asiana flight crashed & August 7, 2013 & August 7, 2013 07:26 & Asiana, flight, Francisco, landed, san \\
\hline 13 & $\begin{array}{l}\text { US military judge refuses to dismiss } \\
\text { charges against Bradley Manning }\end{array}$ & August 18, 2013 & August 18, 2013 14:20 & $\begin{array}{l}\text { Aiding, Bradley, charge, dismiss, enemy, } \\
\text { giving, information }\end{array}$ \\
\hline 14 & $\begin{array}{l}\text { Catherine, the Duchess of Cambridge, } \\
\text { and the royal baby boy }\end{array}$ & August 22, 2013 & August 22, 2013 06:38 & $\begin{array}{l}\text { Baby, birth, boy, Cambridge, duchess, } \\
\text { Kensington, palace }\end{array}$ \\
\hline 15 & Crashed train in Spain & August 25, 2013 & August 25, 2013 10:05 & $\begin{array}{l}\text { 1944, 78, crash, deadliest, death, Galicia, } \\
\text { rises, Spain, toll, train }\end{array}$ \\
\hline 16 & Jewels are stolen in Cannes & August 28, 2013 & August 28, 2013 12:01 & $\begin{array}{l}136 \text { m, } 136 \text { m, Cannes, double, estimate, } \\
\text { French, jewels, stolen, Sunday, worth }\end{array}$ \\
\hline
\end{tabular}

the performance of application system each sampling only collected a number $N$ of messages which the system can process instantly. If messages come so quickly, they can be stored in a cache for next processes. The number of term distributed in a partition will be tracked as a series by time, where time concept is the partition index; the sequence is used to determine another relevant feature of a term or term pairs.

3.2. Topic Trend Detection. Around occurrence timestamp of a new topic, related messages will be fed into the system quickly; we found that these tweets contain some similar keyword or phrase which has strong meaning to a topic's content. Some SNSs restrict the length of posting message; thus people will try to use the most impressive term to describe facts; this is a one reason to make the appearance frequency of it be increased. The significant terms are surely to be used, if the message contains different factst. Besides that, no single keyword or phrase can describe at all a fact; it has to be combined together in a complete sentence; hence the cooccurring frequency of each individual term pair will also be increased. Figure 3 shows an example about timestamp tracking of two terms $w 1$ and $w 2$, where $t_{w 1}, t_{w 2}$ is the first time, $w 1, w 2$ appear and are tracked, and the time range $\left[t_{w 2}, t_{\text {current }}\right]$ is the period where an potential topic trend related to $w 1$ and $w 2$ can occur. Using this feature, we propose 


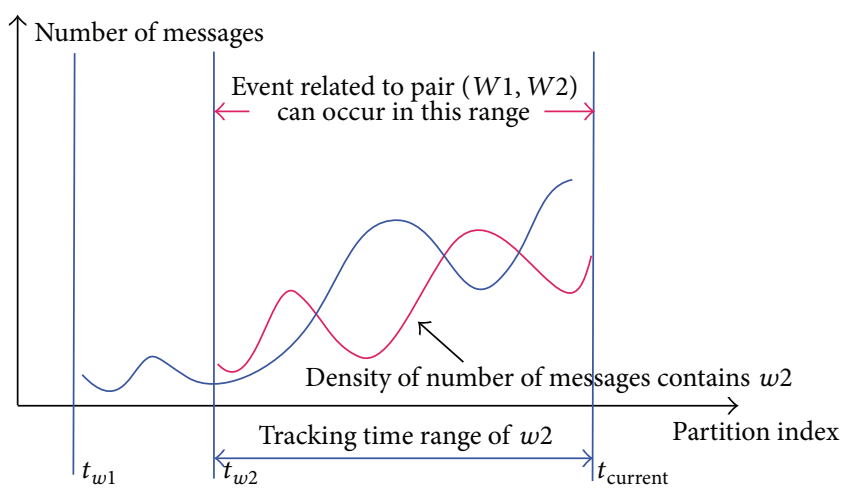

Figure 3: Terms tracking time range.

a method to determine a potential topic trend by creating its characterization set, which is formed by collecting the most cooccurring term pairs, but ensure that each respective pair in the set has a high cooccurring frequency than a threshold. In other words, by detecting sudden changes in the sequence of number of term appearances, we can find when a new topic occurred.

\section{Experimental Result}

For evaluating the clustering method, we unify to specify fixed values: the time interval length of one stream partition $\Delta t$ is 30 minutes; the maximum number of data points in a partition $N$ is not more than 100 items; not any topic trend candidates are tracked more than 2 days from the last appearance of its relevant message. However, we use two different input settings for our system depending on which kind of testing purpose and individual dataset is used. We found that our approach works well on an input dataset which contains a set of strong content-related tweets rather than a group of self-expressing tweets toward a fact. This is suitable for the main goal of this work to detect a trending topic which is drawing more attention from many people with a large enough number of interested news.

We have collected approximately 5 thousand tweets which are posted by Twitter accounts of breaking news agents such as CNN, BBC, and Reuters, from April 01, 2013, to July 31, 2013. The dataset is used to extract potential topic trends; then the result is evaluated by aligning extracted topics with wellknown facts which occur around the time range. In Table 1, we show top 16 topic trends and their time range as an illustration for our experimental result.

\section{Conclusion}

In this paper, we have demonstrated our proposed system to detect topic trend from data stream of certain Twitter accounts. The applied method has advantage to detect new topic trend by clustering related message into corresponding categories using content-based methods and temporal information and propagating information of the messages among social community's members in runtime. The application is useful to help the user to track easily hot topics which are often discussed recently. But speed of performance still is problem; it needs to be improved by reimplementing better algorithms in future.

As a research limitation, we have realized that the communications on social media can be among heterogeneous communities (e.g., multilingual communities). Thus, as a future work, we want to consider the semantic identification methods [17] for the community of practice.

\section{Conflict of Interests}

The authors declare that there is no conflict of interests regarding the publication of this paper.

\section{Acknowledgment}

This work was supported by the National Research Foundation of Korea (NRF) Grant funded by the Korean government (MEST) (no. 2011-0017156) and the BK21+ program of the National Research Foundation (NRF) of Korea.

\section{References}

[1] J. J. Jung, "Semantic preprocessing for mining sensor streams from heterogeneous environments," Expert Systems with Applications, vol. 38, no. 5, pp. 6107-6111, 2011.

[2] H. Sayyadi, M. Hurst, and A. Maykov, "Event detection and tracking in social streams," in Proceedings of the 3rd International Conference on Weblogs and Social Media (ICWSM '09), E. Adar, M. Hurst, T. Finin, N. S. Glance, N. Nicolov, and B. L. Tseng, Eds., pp. 17-20, AAAI Press, San Jose, Calif, USA, May 2009.

[3] T. Brants and F. Chen, "A system for new event detection," in Proceedings of the 26th Annual International ACM SIGIR Conference on Research and Development in Information Retrieval, pp. 330-337, ACM, Toronto, Canada, August 2003.

[4] H. Becker, M. Naaman, and L. Gravano, "Event identification in social media," in Proceedings of the 12th International Workshop on the Web and Databases (WebDB '09), Providence, RI, USA, June 2009.

[5] Q. Zhao, P. Mitra, and B. Chen, "Temporal and information flow based event detection from social text streams," in Proceedings of the 22nd AAAI Conference on Artificial Intelligence, pp. 15011506, AAAI, British Columbia, Canada, July 2007.

[6] D. N. Trung and J. J. Jung, "Sentiment analysis based on fuzzy propagation in online social networks: a case study on TweetScope," Computer Science and Information Systems, vol. 11, no. 1, pp. 215-228, 2014.

[7] S. Zhong, "Efficient streaming text clustering," Neural Networks, vol. 18, no. 5-6, pp. 790-798, 2005.

[8] C. C. Aggarwal and K. Subbian, "Event detection in social streams," in Proceedings of the 12th SIAM International Conference on Data Mining, pp. 624-635, SIAM/Omnipress, Anaheim, Calif, USA, April 2012.

[9] Y. Yang, J. Zhang, J. Carbonell, and C. Jin, “Topic-conditioned novelty detection," in Proceedings of the 8th ACM SIGKDD International Conference on Knowledge Discovery and Data Mining (KDD '02), pp. 688-693, ACM, Alberta, Canada, July 2002. 
[10] R. Kumar, J. Novak, and A. Tomkins, "Structure and evolution of online social networks," in Proceedings of the 12th ACM SIGKDD International Conference on Knowledge Discovery and Data Mining (KDD '06), pp. 611-617, ACM, Philadelphia, Pa, USA, August 2006.

[11] A. Mislove, M. Marcon, P. K. Gummadi, P. Druschel, and B. Bhattacharjee, "Measurement and analysis of online social networks," in Proceedings of the 7th ACM SIGCOMM Conference on Internet Measurement, C. Dovrolis and M. Roughan, Eds., pp. 29-42, ACM, San Diego, Calif, USA, October 2007.

[12] L. Jin, Y. Chen, T. Wang, P. Hui, and A. V. Vasilakos, "Understanding user behavior in online social networks: a survey," IEEE Communications Magazine, vol. 51, no. 9, pp. 144-150, 2013.

[13] A. Kontostathis, L. Galitsky, W. M. Pottenger, S. Roy, and D. J. Phelps, A Survey of Emerging Trend Detection in Textual Data Mining, chapter 9, Springer, New York, NY, USA, 2003.

[14] F. Bonchi, C. Castillo, A. Gionis, and A. Jaimes, "Social network analysis and mining for business applications," ACM Transactions on Intelligent Systems and Technology, vol. 2, no. 3, p. 22, 2011.

[15] Y. Liu, J. Cai, J. Yin, and A. W. Fu, "Clustering text data streams," Journal of Computer Science and Technology, vol. 23, no. 1, pp. 112-128, 2008.

[16] J. G. Dias, "Model selection criteria for model-based clustering of categorical time series data: a monte carlo study," in Proceedings of the 30th Annual Conference of the Gesellschaft für Klassifikation e.V., R. Decker and H. J. Lenz, Eds., vol. 11 of Studies in Classification, Data Analysis, and Knowledge Organization, pp. 23-30, Springer, March 2006.

[17] J. J. Jung, "Cross-lingual query expansion in multilingual folksonomies: a case study on Flickr," Knowledge-Based Systems, vol. 42 , pp. 60-67, 2013. 

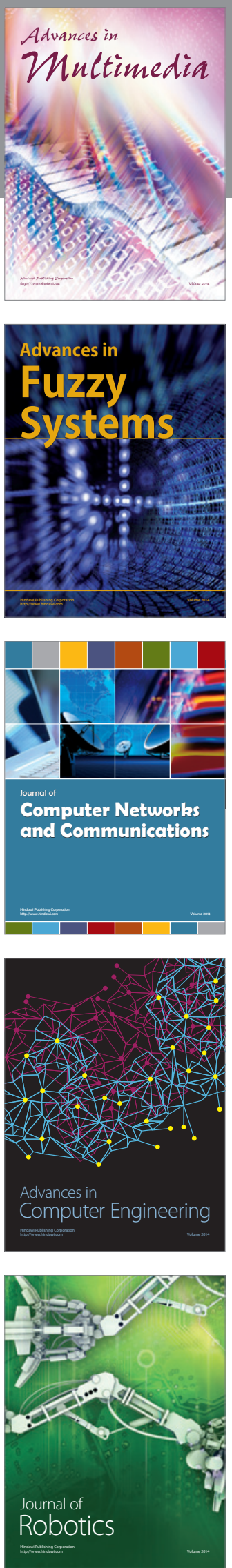

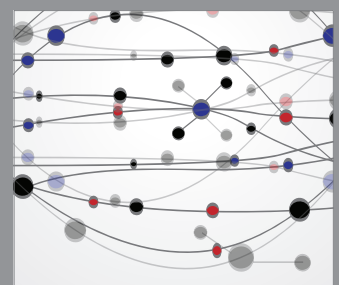

The Scientific World Journal
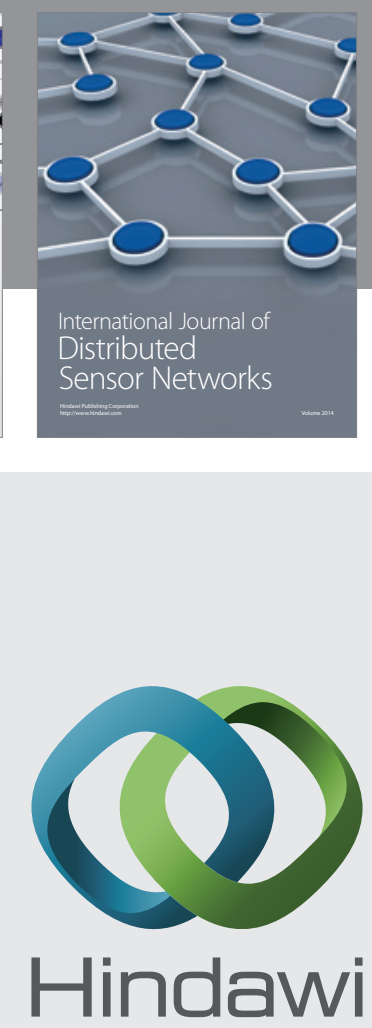

Submit your manuscripts at

http://www.hindawi.com
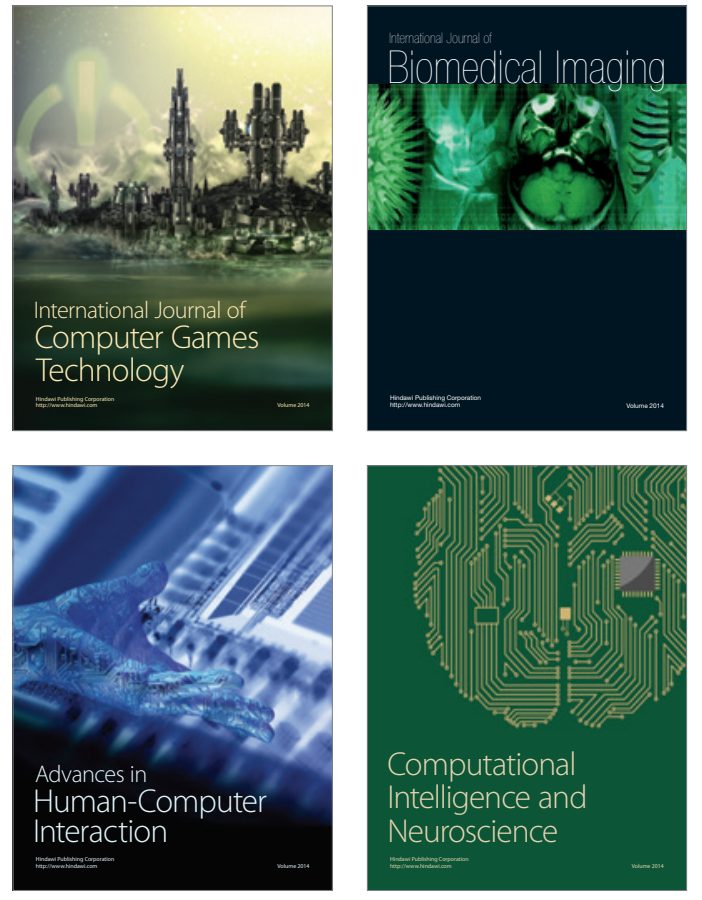
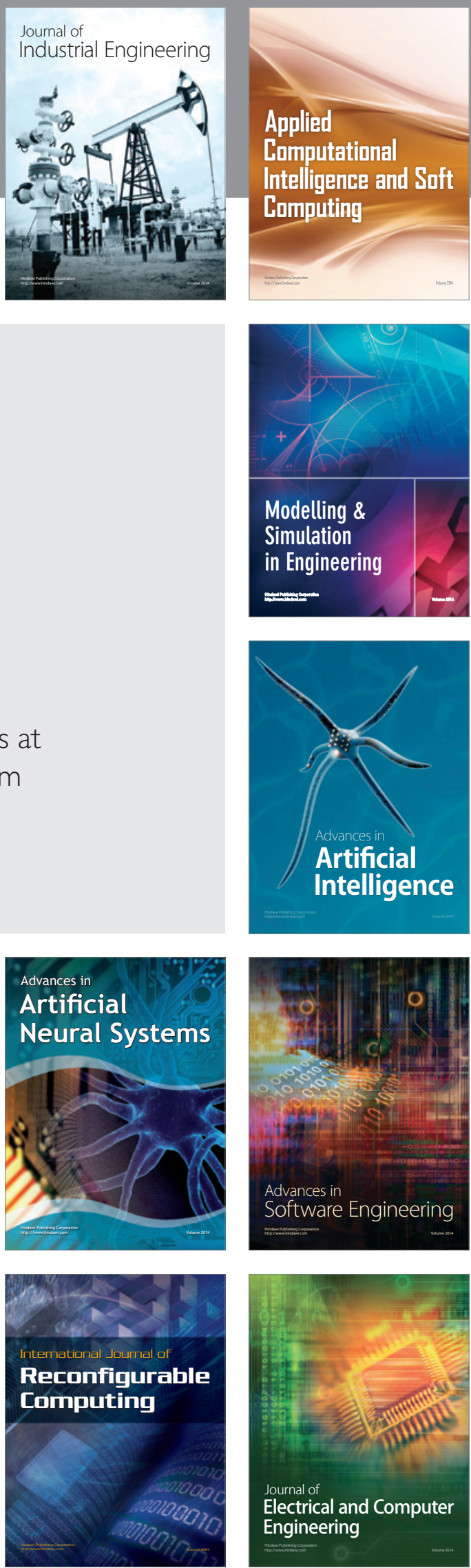